\title{
Design of a Carbon Nanotube/Magnetic Nanoparticle-Based Peroxidase-Like Nanocomplex and Its Application for Highly Efficient Catalytic Oxidation of Phenols
}

\author{
Xiaolei Zuo, Cheng Peng, Qing Huang, Shiping Song, Lihua Wang, Di Li, and Chunhai Fan $(\varangle)$ \\ Shanghai Institute of Applied Physics, Chinese Academy of Sciences, Shanghai 201800, China \\ Received: 30 March 2009 / Revised: 8 May 2009/ Accepted: 19 May 2009 \\ (C)Tsinghua University Press and Springer-Verlag 2009. This article is published with open access at Springerlink.com
}

\begin{abstract}
We report a novel nanotechnology-based approach for the highly efficient catalytic oxidation of phenols and their removal from wastewater. We use a nanocomplex made of multi-walled carbon nanotubes (MWNTs) and magnetic nanoparticles (MNPs). This nanocomplex retains the magnetic properties of individual MNPs and can be effectively separated under an external magnetic field. More importantly, the formation of the nanocomplex enhances the intrinsic peroxidase-like activity of the MNPs that can catalyze the reduction of hydrogen peroxide $\left(\mathrm{H}_{2} \mathrm{O}_{2}\right)$. Significantly, in the presence of $\mathrm{H}_{2} \mathrm{O}_{2}$, this nanocomplex catalyzes the oxidation of phenols with high efficiency, generating insoluble polyaromatic products that can be readily separated from water.
\end{abstract}

\section{KEYWORDS}

Carbon nanotubes, magnetic nanoparticles, phenol, peroxidase, catalysis

\section{Introduction}

Given the global water crisis, it is highly necessary to develop new methods for water clean-up [1,2]. Nanotechnology has become a promising approach toward this goal. Nanomaterials with different compositions, dimensions, and shapes have found applications in a wide range of areas [3-11]. In particular, complexes of nanomaterials not only combine advantages of individual nanomaterials but may lead to novel, otherwise unattainable, properties; these nevertheless have yet to be extensively exploited. Here we demonstrate that magnetic nanoparticles (MNPs) can be readily loaded on the surface of multi-walled carbon nanotubes
(MWNTs), leading to a highly active peroxidaselike nanocomplex that can efficiently treat phenols in water (Fig. 1).

Phenols are the major pollutants in industrial wastewaters. Based on the finding that a natural enzyme, horseradish peroxidase (HRP) polymerizes phenols in plants [12], Klibanov et al. developed an enzyme-based method for water treatment via HRP-catalyzed removal of phenols, which has been regarded as an alternative to conventional chemically-, energetically- and operationallyintensive methods [13]. However, large-scale use of this enzyme-based method in wastewater treatment remains to be explored, partly because of high cost and limited stability of HRP.

Address correspondence to fchh@sinap.ac.cn 


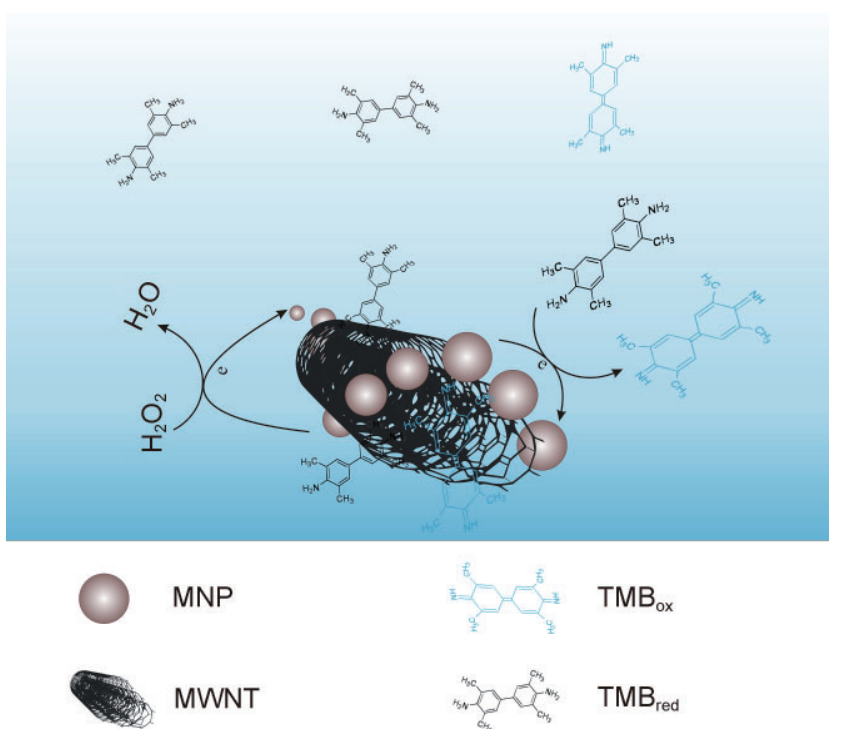

Figure 1 Scheme for the MNP/MWNT nanocomplex-based catalysis. MWNTs serve as a concentrator that confines both MNPs and substrates in the nanoscale geometry, where MNPs catalyze the $\mathrm{H}_{2} \mathrm{O}_{2}$ reduction with enhanced activity

MNPs have proven particularly useful for various applications relying on their superparamagnetic properties [14, 15]. Interestingly, it was recently found that $\mathrm{Fe}_{3} \mathrm{O}_{4}$ MNPs possess intrinsic HRPlike catalytic activity toward the reduction of $\mathrm{H}_{2} \mathrm{O}_{2}$ [16]. This "artificial enzyme" is highly robust, offering unprecedented advantages in constructing peroxidase-like-based bioassays and phenol decomposition $[17,18]$. Carbon nanotubes are a highly popular nanomaterial because of their unique mechanical, electronic, and catalytic properties [1922]. Of particular interest is that the very high surfaceto-volume ratio of nanotubes makes them ideal vectors for the design of functional nanocomplexes. In this paper we report the preparation and properties of a nanocomplex of MNPs and MWNTs.

\section{Experimental}

\subsection{Materials}

Glucose oxidase $\left(\mathrm{GO}_{x}\right)$ was purchased from Vector Laboratories, and horseradish peroxidase (HRP) and 3,3',5,5'-tetramethylbenzidine (TMB) were from Sigma-Aldrich. MWNTs (50 nm in diameter) were obtained from Shenzhen Nanotech Port Co. Ltd (Shenzhen, China). Other reagents were commercially available and of analytical reagent grade. Solutions were prepared with ultrapure water (resistivity of $18 \mathrm{M} \Omega \cdot \mathrm{cm}$ ) from a Millipore Milli-Q water purification system.

\subsection{Pretreatment of MWNTs, chemical synthesis of MNPs and formation of the nanocomplex}

The MWNTs were first suspended in a mixture of concentrated $\mathrm{HNO}_{3}$ and $\mathrm{H}_{2} \mathrm{SO}_{4}(\mathrm{v} / \mathrm{v}, 1: 3)$ which was then heated to $80{ }^{\circ} \mathrm{C}$ for $6 \mathrm{~h}$. The acid-treated MWNTs were centrifuged at 12,000 $\mathrm{g}$ for $30 \mathrm{~min}$, and the precipitate was washed with copious amounts of water. The final solution was centrifuged and the precipitate was dried in vacuum. Thus, obtained MWNTs were readily soluble in water (0.1 wt\%).

MNPs were synthesized in a partial reduction method based on the following equations:

$$
\begin{aligned}
& \mathrm{SO}_{3}{ }^{2-}+2 \mathrm{Fe}^{3+}+\mathrm{H}_{2} \mathrm{O} \rightarrow 2 \mathrm{Fe}^{2+}+\mathrm{SO}_{4}{ }^{2-}+2 \mathrm{H}^{+} \\
& 2 \mathrm{Fe}^{3+}+\mathrm{Fe}^{2+}+8 \mathrm{OH}^{-} \rightarrow \mathrm{Fe}_{3} \mathrm{O}_{4}+4 \mathrm{H}_{2} \mathrm{O}
\end{aligned}
$$

$\mathrm{FeCl}_{3} \cdot 6 \mathrm{H}_{2} \mathrm{O}(12.98 \mathrm{~g})$ was dissolved in $100 \mathrm{~mL}$ of water, and poured in a $500 \mathrm{~mL}$ three-necked flask. Freshly prepared sodium sulfite $(0.16 \mathrm{~mol} / \mathrm{L})$ solution $(50 \mathrm{~mL})$ was slowly added to the flask. After the color of the solution turned from red to yellow, $40 \mathrm{~mL}$ of diluted ammonia ( $24 \mathrm{~mL}$ of concentrated ammonia diluted with $16 \mathrm{~mL}$ of water) was rapidly injected into the flask, with vigorous stirring and under the protection of nitrogen. The reaction was kept at $60^{\circ} \mathrm{C}$ for $30 \mathrm{~min}$, and then at room temperature for $2 \mathrm{~h}$. After completion of the reaction, the black precipitate was collected under an external magnetic field and washed twice with sodium chloride solution and then with pure water. The MNPs were dispersed in $0.01 \mathrm{~mol} / \mathrm{L}$ hydrochloric acid solution with a loading of $0.5 \mathrm{wt} \%$.

The stock solutions of MWNTs and MNPs were diluted by 25 times with Milli-Q water. Then, these two solutions were mixed to form the MNP/MWNT nanocomplex.

\section{3 $\mathrm{H}_{2} \mathrm{O}_{2}$ reduction in the presence of magnetic particles}

In a typical colorimetric assay, aliquots $(50 \mu \mathrm{L})$ of different magnetic particles including micrometersized magnetic particles, silica-coated MNPs, MNPs, and the MNP/MWNT nanocomplex were mixed with $50 \mu \mathrm{L}$ of $\mathrm{TMB}$ stock solution $(5 \mathrm{mmol} / \mathrm{L})$ with 2 
$\mathrm{mmol} / \mathrm{L} \mathrm{H}_{2} \mathrm{O}_{2}$ in plastic tubes.

Kinetic assays were performed in 96-well plate at $40{ }^{\circ} \mathrm{C}$. Aliquots $(50 \mu \mathrm{L})$ of either nanocomplex or MNPs (50-fold dilution) were mixed with $50 \mu \mathrm{L}$ of TMB $(5 \mathrm{mmol} / \mathrm{L})$ and $50 \mu \mathrm{L}$ of $\mathrm{H}_{2} \mathrm{O}_{2}$ of a series of concentrations. The absorbance was monitored at 650 $\mathrm{nm}$ using a Tecan microplate reader for $5 \mathrm{~min}$. The Michaelis-Menten constant was calculated based on the Lineweaver-Burk plot: $1 / v=K_{\mathrm{m}} / V_{\max } \cdot C+1 / V_{\max }$ where $v$ is the initial velocity, $V_{\max }$ is the maximal reaction velocity, and $C$ is the concentration of hydrogen peroxide.

In a glucose assay, glucose solutions of appropriate concentration $(50 \mu \mathrm{L})$ and the glucose oxidase solution $(50 \mu \mathrm{L}$ ) were mixed and incubated for $1 \mathrm{~min}$, and then $50 \mu \mathrm{L}$ of TMB solution $(1.6 \mathrm{mmol} / \mathrm{L})$ and $50 \mu \mathrm{L}$ of nanocomplex were added. The assay was performed in a 96-well plate at $25^{\circ} \mathrm{C}$, with the absorbance monitored at $650 \mathrm{~nm}$.

\subsection{Transmission electron microscopy characterization}

Transmission electron microscopy (TEM) samples were prepared by drop casting the MNPs solution or MNP / MWNT nanocomplex solution onto carboncoated copper grids, with excess solvent evaporated. The TEM images were recorded using an FEI/Philips Tecnai G2 Bio TWIN TEM.

\subsection{The X-ray diffraction analysis}

The X-ray diffraction (XRD) analysis was performed with D/Max-2000 XRD diffractometer. The MNPs, MWNTs, and MNP/MWNT nanocomplex were separated from the solution and extensively washed with Milli-Q water. The samples were dried in vacuum before XRD patterns were recorded.

\subsection{FTIR measurement}

FTIR measurements were performed with a PerkinElmer Spectrum ${ }^{\mathrm{TM}} 100$ FTIR spectrometer for samples of MNPs, MWNTs and MNP/MWNT nanocomplexes.

\subsection{Nanocomplex-assisted phenol removal}

Phenol degradation was conducted in a plastic tube at room temperature. In each tube, the nanocomplex was mixed with $500 \mathrm{mmol} / \mathrm{L} \mathrm{H}_{2} \mathrm{O}_{2}$ and phenol of various concentrations. At each checkpoint, the solution was magnetically separated and the supernatant was analyzed by UV-Vis spectrophotometry (U-3010 spectrophotometer, Hitachi).

The phenol concentration was determined based on Emerson's method [23]. A series of $200 \mu \mathrm{L}$ aliquots of the above mentioned supernatants were added to wells of a 96-well plate. Then, $1 \mu \mathrm{L}$ of ammonia was added to each well and mixed. Finally, $1 \mu \mathrm{L}$ of 4 -aminoantipyrine solution (2\%) and $1 \mu \mathrm{L}$ of potassium ferricyanide $(8 \%)$ were added to the wells and mixed. The 96-well plate was incubated in the dark for $10 \mathrm{~min}$. The absorbance at $510 \mathrm{~nm}$ of each well was recorded using a Tecan microplate reader. The "removal efficiency" is defined as the percentage of phenol removed from solution under a given set of conditions.

\section{Results and discussion}

We employed water-soluble (OH-terminated surface) MNPs of $\sim 15 \mathrm{~nm}$ diameter, and found that they were readily adsorbed on the surface of watersoluble carboxylic-functionalized MWNTs, leading to a well dispersed MNP / MWNT nanocomplex in aqueous solution. TEM images of MNPs (Fig. 2(a)), MWNTs (Fig. 2(b)) and the MNP/MWNT nanocomplex (Fig. 2(c)) showed that the MNPs were located on the surface rather than inside the MWNTs. FTIR measurements further confirmed the existence of the MNP/MWNT nanocomplex (Fig. 2(d)). This nanocomplex was very stable in solution for months. Under an external magnetic field, the nanocomplex could be readily separated from the bulk solution within seconds (Fig. 3).

Consistent with the previous report [16], MNPs could catalyze the reduction of $\mathrm{H}_{2} \mathrm{O}_{2}$, instantly leading to a blue color upon the addition of $\mathrm{H}_{2} \mathrm{O}_{2}$ and a co-substrate TMB, suggesting the HRP-like catalytic property of MNPs (Fig. 4). This catalytic activity is critically dependent on the particle size. In contrast to nanometer-sized MNPs, micrometer-sized magnetic particles did not show observable enzymatic-like activity. Also, silica-coated MNPs showed negligible enzymatic-like activity, suggesting that the presence of surface-confined iron was critical for the catalytic reaction. 


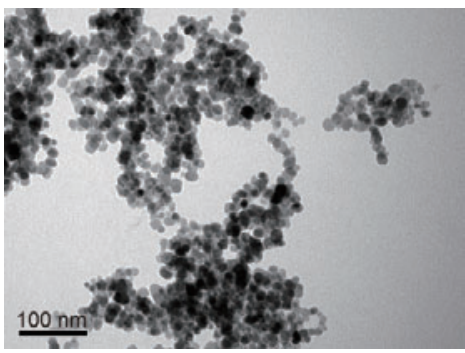

(a)

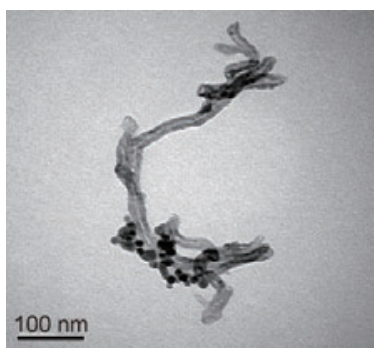

(c)

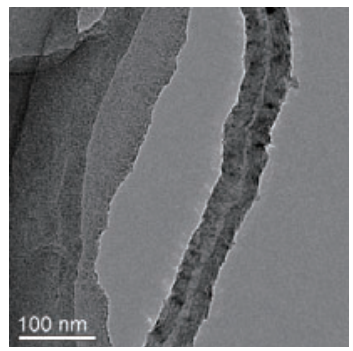

(b)

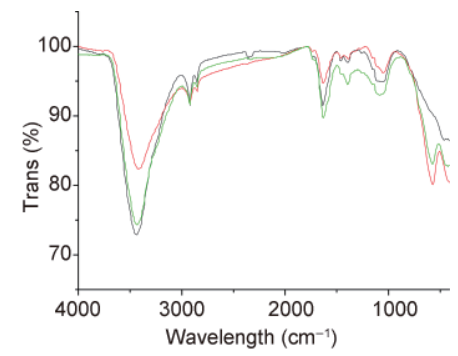

(d)
Figure 2 TEM images of (a) MNPs, (b) MWNTs, and (c) MNPs/ MWNTs nanocomplex, (d) FTIR spectra for MWNTs (black), MNPs (red), and MNPs/MWNTs nanocomplex (green)

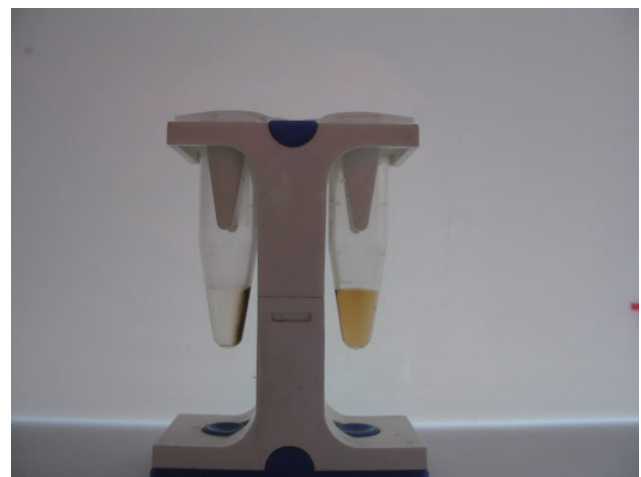

Figure 3 Magnetic separation of MNPs (left) and MNP/MWNT nanocomplex (right). Both can be easily magnetically separated under the external magnet (between the two tubes). As shown in the figure, however, the MNP/MWNT nanocomplex is more rapidly separated (within $30 \mathrm{~s}$ ) while MNPs are not separated within this short period. This is possibly because the particle sizes of the nanocomplex are significantly larger

Interestingly, we found that the activity of MNPs was significantly enhanced upon the formation of the MNP/MWNT nanocomplex. Importantly, the color change for the nanocomplex solution was much faster, and led to a more intense blue color than that with MNPs alone. We then performed kinetic studies to quantitatively evaluate the catalytic ability of this peroxidase-like nanocomplex. As shown in Fig. 4, the nanocomplex-based catalysis was kinetically faster than the MNPs-based one. While the activity of the nanocomplex is still lower than that of the natural HRP enzyme, it is much more active than MNP alone, with the maximum reaction rate $\left(V_{\max }\right)$ increased by over four times, and the apparent Michaelis-Menten constant $\left(K_{\mathrm{m}}\right)$ value decreased by half (Table 1$)$. The activity of the nanocomplex showed a concentration dependence on the concentration of $\mathrm{H}_{2} \mathrm{O}_{2}$ (Fig. 5(a)).

Table 1 Comparison of values of apparent Michaelis-Menten constant $\left(K_{\mathrm{m}}\right)$ and maximum reaction rate $\left(V_{\text {max }}\right)$ for different catalysts

\begin{tabular}{c|c|c}
\hline Substance & $K_{\mathrm{m}}(\mathrm{mmol} / \mathrm{L})$ & $V_{\max }\left(10^{-8} \mathrm{~mol} \cdot \mathrm{L}^{-1} \cdot \mathrm{s}^{-1}\right)$ \\
\hline MNP & $1.13 \pm 0.30$ & $5.0 \pm 0.3$ \\
\hline Nanocomplex & $0.55 \pm 0.17$ & $23 \pm 0.5$ \\
\hline HRP & $0.06 \pm 0.01$ & $15 \pm 0.6$ \\
\hline
\end{tabular}

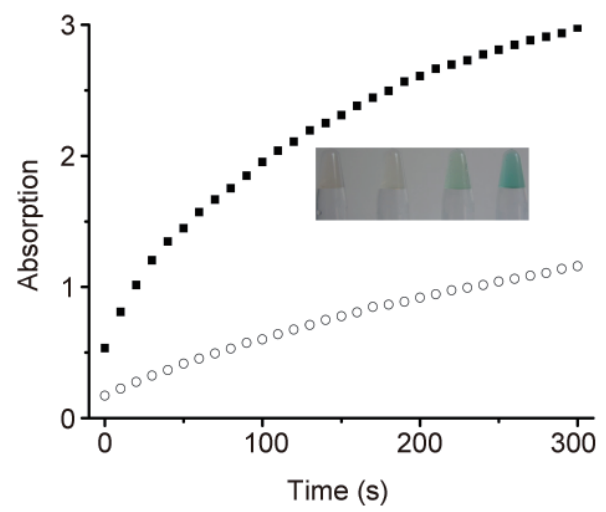

Figure 4 Michaelis-Menten kinetics of the MNPs (open circles) and the nanocomplex (filled squares) in the presence of $1.6 \mathrm{mmol} / \mathrm{L} \mathrm{TMB}$ and $3 \mathrm{mmol} / \mathrm{L} \mathrm{H}_{2} \mathrm{O}_{2}$. Inset: Catalyzed $\mathrm{H}_{2} \mathrm{O}_{2}$ reduction in the presence of TMB. From left to right, micro-sized magnetic particles; silicacoated MNPs; bare MNPs; MNP/MWNT nanocomplex

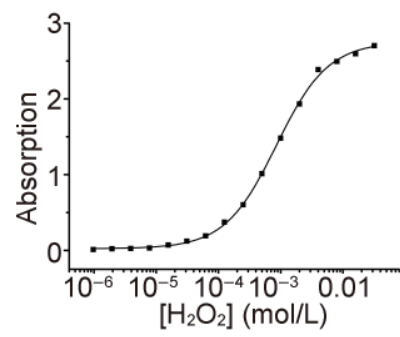

(a)

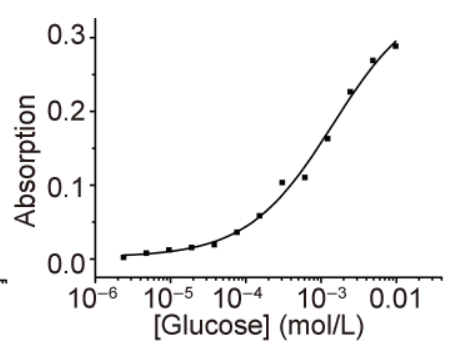

(b)
Figure 5 (a) Plot of $\mathrm{H}_{2} \mathrm{O}_{2}$ concentration versus the absorbance at $650 \mathrm{~nm}$ for the nanocomplex-based catalysis; (b) Plot of glucose concentration versus the absorbance at $650 \mathrm{~nm}$ for a nanocomplex/ $\mathrm{GO}_{x}$-based glucose assay

We also tested the use of the nanocomplex in a coupled enzymatic reaction involving both HRP and glucose oxidase $\left(\mathrm{GO}_{x}\right)$. In a conventional coupled reaction, $\mathrm{GO}_{x}$ catalytically oxidizes glucose to gluconic acid, while in the meantime the substrate 
oxygen is converted to $\mathrm{H}_{2} \mathrm{O}_{2}$. This $\mathrm{H}_{2} \mathrm{O}_{2}$ product can be catalytically reduced by HRP in the presence of TMB co-substrate, leading to the characteristic blue color.

$$
\begin{gathered}
\text { Glucose }+\mathrm{O}_{2} \stackrel{\mathrm{GO}_{x}}{\longrightarrow} \text { Gluconic acid }+\mathrm{H}_{2} \mathrm{O}_{2} \\
\mathrm{HRP}_{\text {red }}+\mathrm{H}_{2} \mathrm{O}_{2} \longrightarrow \mathrm{HRP}_{\mathrm{OX}}+\mathrm{H}_{2} \mathrm{O}
\end{gathered}
$$

When we replaced HRP with the nanocomplex in this coupled reaction, we found that the characteristic color change similarly occurred upon being exposed to glucose. Indeed, when we monitored the absorbance at $650 \mathrm{~nm}$, we found that it was a function of the concentration of glucose in the range $2-10 \mathrm{mmol} / \mathrm{L}$ (Fig. 5(b)). This experiment suggests that the nanocomplex can replace the natural HRP, and remain functional in real peroxidase-based applications.

We ascribe the activity enhancement of the nanocomplex relative to MNPs alone (Fig. 4 and Table 1) to the concentrator effect of MWNTs. MWNTs possess high surface-to-volume ratios as well as high affinity for hydrophobic molecules like TMB, which might increase the local concentration of the substrate and improve the catalytic effect. Indeed, when we added the nanocomplex to a TMB solution and then removed it magnetically, the remaining solution did not show the characteristic blue color upon the addition of HRP (data not shown), suggesting the nanocomplex attracts TMB on its surface and possesses high adsorption affinity for TMB. It is well known that the extraordinarily high catalytic efficiency of natural enzymes arises largely from the ability to bring substrates into proximity with their active sites [24]. The combined effects of MNPs and MWNTs in the nanocomplex resemble those in a real enzyme, that is, the nanocomplex confines both catalyzing species (MNPs) and the substrates in a nanoscale geometry (MWNTs), and the electron communication between MNPs and substrates is electrically "wired" due to the high electrical conductance of MWNTs [25]. These synergetic effects might lead to the observed high catalyzing activity.

We then tested the applicability of the nanocomplex for catalytic treatment of phenol-containing water. HRP oxidizes phenols to phenoxy radicals that polymerize phenols into insoluble polyaromatic products $[13,26]$. When the nanocomplex, $\mathrm{H}_{2} \mathrm{O}_{2}$ and phenol were mixed, the solution readily turned dark brown (Fig. 6), a phenomenon resembling that observed with HRP $[13,26]$. We ascribe this phenol oxidation to a similar mechanism to that reported for HRP-mediated phenol degradation [26], which is shown as follows (" $\mathrm{m}$ " stands for an activated state of the nanocomplex, $\mathrm{Ph}^{*}$ for the phenoxy radical and $\mathrm{Ph}-\mathrm{Ph}$ for the dimeric product).

$$
\begin{aligned}
\text { Nanocomplex }+\mathrm{H}_{2} \mathrm{O}_{2} & \rightarrow \text { Nanocomplex }(\mathrm{m}) \\
\text { Nanocomplex }(\mathrm{m})+\mathrm{Ph} & \rightarrow \text { Nanocomplex }+\mathrm{Ph}^{\bullet} \\
\mathrm{Ph}^{\bullet}+\mathrm{Ph}^{\bullet} & \rightarrow \mathrm{Ph}-\mathrm{Ph}
\end{aligned}
$$

Although, unlike HRP, the nanocomplex itself has a light brown color, the color change was still clearly visible. This efficient phenol degradation can be ascribed to the catalytic properties of the nanocomplex, and the absence of leaching of iron ions. Indeed, when we magnetically separated the nanocomplex, the supernatant did not show significant catalysis as no visual color change was observed.

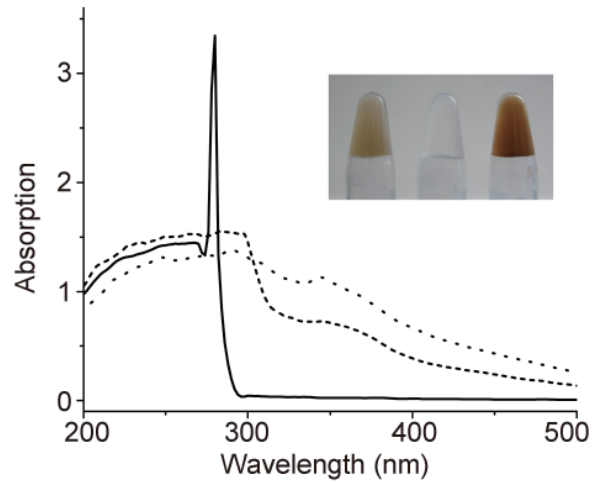

(a)

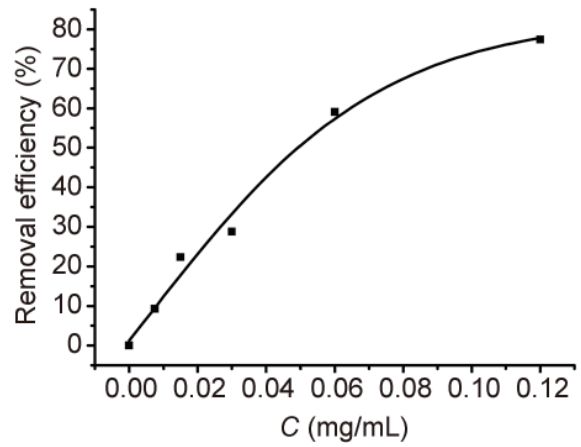

(b)

Figure 6 (a) UV monitoring of phenol treatment: $0 \mathrm{~h}$ (solid line); $0.5 \mathrm{~h}$ (dashed line); $2 \mathrm{~h}$ (dotted line). Inset: Photographs of the phenol treatment. Nanocomplex alone (left), $\mathrm{H}_{2} \mathrm{O}_{2}$ alone (center), and mixture of nanocomplex and $\mathrm{H}_{2} \mathrm{O}_{2}$ (right). (b) Variation in removal efficiency for phenol ( $0.25 \mathrm{~g} / \mathrm{L}, 30 \mathrm{~min})$ with the concentration of nanocomplex 
We further studied the phenol removal efficiency by $\mathrm{UV}-\mathrm{Vis}$ monitoring at $280 \mathrm{~nm}$ [23]. After treatment with the nanocomplex and $\mathrm{H}_{2} \mathrm{O}_{2}$, the characteristic peak at $280 \mathrm{~nm}$ for phenol disappeared within $30 \mathrm{~min}$ and a new broad peak appeared at $\sim 370 \mathrm{~nm}$ (Fig. $6(\mathrm{a}))$, corresponding to the absorbance of oxidation products. This new peak increased gradually with time $(2 \mathrm{~h})$, suggesting higher polymerization degree with elongated treatment time. The removal efficiency increased with nanocomplex concentration in the range $0.01-0.12 \mathrm{mg} / \mathrm{mL}$ (Fig. 6(b)). Within a short-period

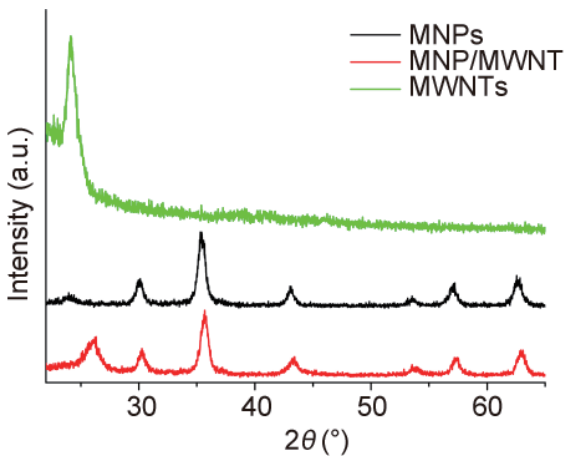

(a)

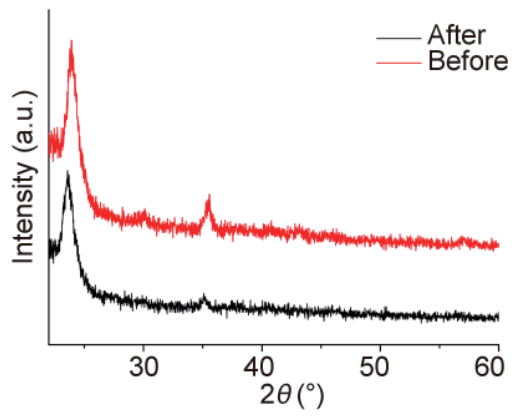

(b)

Figure 7 (a) XRD patterns of MNPs, MWNTs and MNPs/MWNTs nanocomplex. MWNTs are characterized by a peak at $24^{\circ}, 2 \theta$, while MNPs give a peak at $36^{\circ}, 2 \theta$. The MNPs/MWNTs nanocomplex possessess both peaks, confirming the formation of the complex. (b) XRD analysis of the MNPs/MWNTs nanocomplex before and after phenol oxidation catalysis. After the nanocomplex was employed for catalysis, both peaks were retained, suggesting that the nanocomplex was still intact. The peak at $36^{\circ}, 2 \theta$ decreased in intensity however, implying that MNPs were partially oxidatively degraded during the catalysis. Of note, MNPs were of 50-fold excess in (a) as compared to MWNTs in this XRD measurement to obtain high resolution XRD; while MNPs:MWNTs=5:1 in (b) to mimic the real catalysis conditions treatment (30 $\mathrm{min})$, the removal efficiency of phenol $(0.25 \mathrm{~g} / \mathrm{L})$ was as high as $\sim 80 \%$ with $0.12 \mathrm{mg} / \mathrm{L}$ nanocomplex. The phenol removal efficiency could reach $\sim 99 \%$ after a prolonged treatment time $(10 \mathrm{~h})$. This efficiency was slightly lower for a higher concentration of phenol of $1 \mathrm{~g} / \mathrm{L}$ ( 97\%). These data show that the nanocomplex serves as a highly efficient catalyst for the treatment of phenol-containing water.

After the treatment of phenol-containing water, both the catalytically formed products and the nanocomplex could be easily separated. The resulting dark-brown colored polyaromatic product could be separated from the solution by sedimentation and filtration. Under an external magnetic field, the nanocomplex could be magnetically separated from the solution. We found that this separated nanocomplex could still catalyze the oxidation of phenols, leading to a dark-brown mixture. The recycled nanocomplex retained $\sim 60 \%$ of its catalytic activity (i.e., removal efficiency decreased by $\sim 40 \%$ ). The activity loss is possibly due to the oxidative degradation of MNPs (as also confirmed by XRD analysis, Fig.7).

\section{Conclusions}

We have demonstrated that MNPs are readily adsorbed at the surface of MWNTs, forming a nanocomplex that resembles natural HRP and can efficiently treat phenol-containing wastewater. This nanocomplex has several attractive features. First, the nanocomplex formation enhances the intrinsic peroxidase-like activity of MNPs, making it a highly efficient catalyst for the oxidation of phenols. Second, the nanocomplex retains the superparamagnetic property of MNPs, and thus it can be easily separated from treated water under a magnetic force. Third, the separated nanocomplex is reusable, which may reduce the cost for water treatment. Finally, compared to natural HRP, this inorganic nanocomplex is chemically stable and can be mass-produced. Given the high efficiency, low cost, easy operation, high stability and reusability offered by this novel nanocomplex, we expect that it might be a promising tool in the area of wastewater treatment.

\section{Acknowledgements}

We thank Prof. Jun Hu for helpful discussion. This work was supported by the National Natural Science Foundation (20873175 and 20725516), the Shanghai Municipal Commission for Science and Technology (0752nm021), and the Ministry of Science and 
Technology of China (2006CB933000, 2007CB936000, and 2007AA06A406).

\section{References}

[1] Shannon, M. A.; Bohn, P. W.; Elimelech, M.; Georgiadis, J. G.; Marinas, B. J.; Mayes, A. M. Science and technology for water purification in the coming decades. Nature 2008, 452, 301-310.

[2] Editorial: Cleaning up water. Nat. Mater. 2008, 7, 341.

[3] Gupta, A. K.; Gupta, M. Synthesis and surface engineering of iron oxide nanoparticles for biomedical applications. Biomaterials 2005, 26, 3995-4021.

[4] Su, Y. Y.; He, Y.; Lu, H. T.; Sai, L. M.; Li, Q. N.; Li, W. X.; Wang, L. H.; Shen, P. P.; Huang, Q.; Fan, C. H. The cytotoxicity of cadmium-based, aqueous phasesynthesized, quantum dots and its modulation by surface coating. Biomaterials 2009, 30, 19-25.

[5] Rosi, N. L.; Mirkin, C. A. Nanostructures in biodiagnostics. Chem. Rev. 2005, 105, 1547-1562.

[6] Lu, W.; Lieber, C. M. Nanoelectronics from the bottom up. Nat. Mater. 2007, 6, 841-850.

[7] Chan, C. K.; Peng, H. L.; Liu, G.; Mcllwrath, K.; Zhang, X. F.; Huggins, R. A.; Cui, Y. High performance lithium battery anodes using silicon nanowires. Nat. Nanotech. 2008, 3, 31-35.

[8] Nie, S. M.; Xing, Y.; Kim, G. J.; Simons, J. W. Nanotechnology applications in cancer. Annu. Rev. Biomed. Eng. 2007, 9, 257-288.

[9] Hochbaum, A. I.; Chen, R. K.; Delgado, R. D.; Liang, W. J.; Garnett, E. C.; Najarian, M.; Majumdar, A.; Yang, P. D. Enhanced thermoelectric performance of rough silicon nanowires. Nature 2008, 451, 163-168.

[10] Ma, D. D. D.; Lee, C. S.; Au, F. C. K.; Tong, S. Y.; Lee, S. T. Small-diameter silicon nanowire surfaces. Science 2003, 299, 1874-1877.

[11] Alivisatos, P. The use of nanocrystals in biological detection. Nat. Biotechnol. 2004, 22, 47-52.

[12] Freudenberg, K.; Neish, A. C. Constitution and Biosynthesis of Lignin; Springer: New York, 1968.

[13] Klibanov, A. M.; Tu, T. M.; Scott, K. P. Peroxidasecatalyzed removal of phenols from coal-conversion waste-waters. Science 1983, 221, 259-260.

[14] Lim, Y. T.; Cho, M. Y.; Lee, J. M.; Chung, S. J.; Chung,
B. H. Simultaneous intracellular delivery of targeting antibodies and functional nanoparticles with engineered protein G system. Biomaterials 2009, 30, 1197-1204.

[15] Chertok, B.; Moffat, B. A.; David, A. E.; Yu, F. Q.; Bergemann, C.; Ross, B. D.; Yang, V. C. Iron oxide nanoparticles as a drug delivery vehicle for MRI monitored magnetic targeting of brain tumors. Biomaterials 2008, 29, 487-496.

[16] Gao, L. Z.; Zhuang, J.; Nie, L.; Zhang, J. B.; Zhang, Y.; Gu, N.; Wang, T. H.; Feng, J.; Yang, D. L.; Perrett, S.; Yan, $\mathrm{X}$. Intrinsic peroxidase-like activity of ferromagnetic nanoparticles. Nat. Nanotechnol. 2007, 2, 577-583.

[17] Ho, M. Y. K.; Rethnitz, G. A. Highly stable biosensor using an artificial enzyme. Anal. Chem. 1987, 59, 536537.

[18] Zhang, J. B.; Zhuang, J.; Gao, L. Z.; Zhang, Y.; Gu, N.; Feng, J.; Yang, D. L.; Zhu, J. D.; Yan, X. Y. Decomposing phenol by the hidden talent of ferromagnetic nanoparticles. Chemosphere 2008, 73, 1524-1528.

[19] lijima, S. Helical microtubules of graphitic carbon. Nature 1991, 354, 56-58.

[20] Cao, A. Y.; Dickrell, P. L.; Sawyer, W. G.; GhasemiNejhad, M. N.; Ajayan, P. M. Super-compressible foamlike carbon nanotube films. Science 2005, 310, 1307-1310.

[21] Ajayan, P. M.; Tour, J. M. Materials science-Nanotube composites. Nature 2007, 447, 1066-1068.

[22] Chen, F. M.; Wang, B.; Chen, Y.; Li, L. J. Towards the extraction of single species of single-walled carbon nanotubes using fluorene-based polymers. Nano Lett. 2007, 7, 3013-3017.

[23] Emerson, E. The condensation of aminoantipyrine. II. A new color test for phenolic compounds. J. Org. Chem. 1943, 8, 417-428.

[24] Ringe, D.; Petsko, G. A. How enzymes work? Science 2008, 320, 1428-1429.

[25] McDonald, T. J.; Svedruzic, D.; Kim, Y. H.; Blackburn, J. L.; Zhang, S. B.; King, P. W.; Heben, M. J. Wiring-up hydrogenase with single-walled carbon nanotubes. Nano Lett. 2007, 7, 3528-3534.

[26] Yu, J.; Taylor, K. E.; Zou, H. X.; Biswas, N.; Bewtra, J. $K$. Phenol conversion and dimeric intermediates in horseradish peroxidase-catalyzed phenol removal from water. Environ. Sci. Technol. 1994, 28, 2154-2160. 